\title{
Investigation of the binary fraction among candidate A-F type hybrid stars detected by Kepler
}

\author{
P. Lampens ${ }^{1, a}$, Zs. Bognár ${ }^{2}$, Y. Frémat ${ }^{1}$, Á. Sódor $^{1,2}$, L. Vermeylen $^{1}$, R. De Nutte ${ }^{3}$, R. Lombaert ${ }^{3}$, and \\ P. De Cat ${ }^{1}$
}

1 Koninklijke Sterrenwacht van België, Brussel, Belgium

2 Konkoly Observatory, MTA CSFK, Budapest, Hungary

3 Instituut voor Sterrenkunde, K.U. Leuven, Leuven, Belgium

\begin{abstract}
We are currently monitoring up to 40 Kepler candidate $\delta$ Scuti- $\gamma$ Doradus (resp. $\gamma$ Doradus- $\delta$ Scuti) hybrid stars in radial velocity in order to identify the physical cause behind the low frequencies observed in the periodograms based on the ultra-high accuracy Kepler space photometry. The presence of low frequency variability in unevolved or slightly evolved oscillating A/F-type stars can generally be explained in three ways: either 1) the star is an (un)detected binary or multiple system, or 2) the star is a g-mode pulsator (i.e. a genuine hybrid), or 3) the star's atmosphere displays an asymmetric intensity distribution (caused by spots, i.e. chemical anomalies, or by (very) high rotation), which is detected through rotational modulation. Our targets were selected from the globally characterized variable A/F-type stars of the Kepler mission [7]. We observe each star at least 4 times unevenly spread over a time lapse up to 2 months with the HERMES spectrograph [6]. In the case of composite, multiple-lined spectra, these observations also provide the atmospheric properties of each component. Our principal goal is to estimate the fraction of short-period, spectroscopic systems in the sample.
\end{abstract}

\section{Introduction}

Among the latest discoveries regarding A-F type stars, the detection of many candidate pulsating hybrid stars based on the analysis of CoRoT and Kepler data is surprising. These candidate hybrid stars are variable A- and F-type stars located across the full width of the instability strips of both the $\delta$ Sct and the $\gamma$ Dor pulsators. Their light curves exhibit variability in two different regimes [2,7]. We are addressing the question of the physical mechanism responsible for the low frequencies observed in their periodograms, especially because we don't know their true binary fraction. We thus initiated a programme to collect complementary spectroscopy for a sample of 40 brighter candidate targets [7]. These observations are conducted in the high-resolution mode $(\mathrm{R} \sim 85.000)$ with the échelle spectrograph HERMES attached to the 1.2-m Mercator telescope at the observatory Roque de los Muchachos (La Palma, Spain) [6].

\section{Observations and methodology}

Our objective is a) to search for a possible signature of binarity (resp. multiplicity) in the spectra, and b) to determine improved atmospheric parameters as well as $v \sin i$ in order to locate the target or its

a e-mail: patricia.lampens@oma.be Based on data gathered with NASA's Discovery mission Kepler. Also based on data gathered with the HERMES spectrograph, installed at the Mercator Telescope, operated by the Institute of Astronomy, K.U.Leuven, Leuven, on the island of La Palma at the Spanish Observatorio del Roque de los Muchachos of the Instituto de Astrofísica de Canarias.

This is an Open Access article distributed under the terms of the Creative Commons Attribution License 4.0, which permits unrestricted use, distribution, and reproduction in any medium, provided the original work is properly cited. 

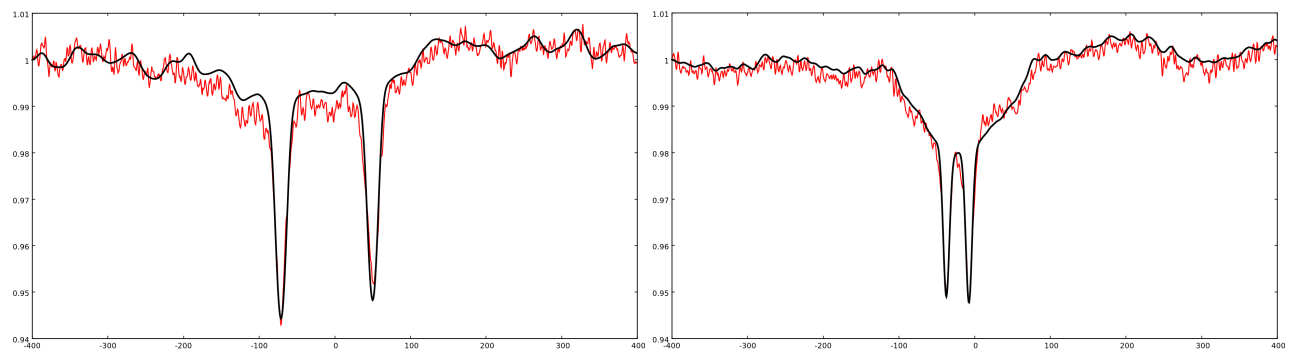

Fig. 1. Left: Observed (red) versus modelled CCF (black) for KIC 4480321 and a model consisting of an (A5+F0+F0) triple system of $v \sin i=(160,10,10) \mathrm{km} \mathrm{s}^{-1}$. Right: Observed versus modelled CCF for KIC 6381306 and a model of an (A5+A7+A7) triple system of $v \sin i=(90,0,0) \mathrm{km} \mathrm{s}^{-1}$.

components more accurately in the H-R diagram. Each target is repeatedly but unevenly observed at 4 different epochs at least. Our échelle spectra cover time scales of days-weeks up to about 2 months, sometimes even longer. We aim to achieve a precision of about $1 \mathrm{~km} \mathrm{~s}^{-1}$ per observation. The radial velocities are obtained applying the cross-correlation (CC) technique using masks of appropriate type on the reduced, normalized spectra. In the case of a binary system, we use a 2D cross-correlation method following a procedure described by [5]. We also explore the parameter space in terms of spectral class and $v \sin i$ and adopt the best fitting set depending on the smallest sum of the squared residuals in the sense (observed - synthetic) spectra.

\section{Discussion}

Through the detection of line multiplicity or from evidence of significant variations in the radial velocity data, we identified two new triple systems (cf. Fig. 1) and a double-lined spectroscopic binary (KIC 8975515). The changes observed in the cross-correlation functions of KIC 6951642 and KIC 7119530 are indicative of line profile variations probably associated with non-radial pulsations. The cross-correlation functions of KIC 11602449, on the other hand, look perfectly stable. It shows that we have to be careful with the conclusions about hybrid pulsation in this part of the H-R diagram. The surface deformations arising from tides in close, eccentric binaries might explain (some of the) detected low frequencies (e.g. [3]). Another possible mechanism is rotational modulation (e.g. [1]).

\section{Conclusion}

Our programme represents a first step in the investigation of the new A/F-type variability phenomenon. In 9 cases at least, multiplicity is clearly detected. We aim to estimate the fraction of short-period spectroscopic systems (i.e. with periods between about 1 and 50 days) among the selected candidate stars. After identification of the most obvious binary/multiple systems and relocation in the H-R diagram thanks to the improved atmospheric parameters, we will be able to identify more precisely the parameter region where hybrid pulsation is mostly occurring.

\section{References}

1. Balona, L. A., Breger, M. Catanzaro, G. et al., MNRAS 424, (2012) 1187-1196

2. Grigahcène, A., Antoci, V., Balona, L., et al., ApJ 713, (2010) L192

3. Hareter, M., Paparó, M., Weiss, W. et al., A\&A 567, (2014), A124

4. Lampens, P., Tkachenko, A., Lehmann, H. et al., A\&A 549, (2013) A104

5. Mazeh, T., Zucker, S., Ap\&SS 212, (1994) 349-356

6. Raskin, G., Van Winckel, H., Hensberge, H., et al., A\&A 526, (2011) A69

7. Uytterhoeven, K., Moya, A., Grigahcène, A., et al., A\&A 543, (2011) A125 